\title{
APGAR score - the most accurate indicator for the risk of neonatal infection, correlated with the number of days of hospitalization and parity
}

\author{
Titiana Cornelia Cotoi ${ }^{1}$, Raluca Niculescu², Adrian-Horatiu Sabau'², \\ Mihaela Cornelia Sincu², Diana Chiorean², Laura Mihaela Suciü3 ${ }^{3}$ Daniela Lucia Muntean ${ }^{4}$ \\ ${ }^{1}$ IOSUD, "George Emil Palade" University of Medicine, Pharmacy, Science and Technology, \\ Tg. Mures, Romania \\ ${ }^{2}$ Department of Pathology, Mures County Clinical Hospital, Tg. Mures, Romania \\ ${ }^{3}$ Neonatology Clinic, "George Emil Palade" University of Medicine, Pharmacy, Science and Technology, \\ Tg. Mures, Romania \\ ${ }^{4}$ Faculty of Pharmacy, "George Emil Palade" University of Medicine, Pharmacy, Science and Technology, \\ Tg. Mures, Romania
}

\begin{abstract}
Introduction. Infections in newborns are a widely studied topic worldwide. Neonatal infections are of two types: those with early onset, acquired from microorganisms from the female genital tract or peripartum and those with late onset, which appeared as a result of invasive procedures, due to nosocomial or invasive germs peri- or postpartum.

Objectives. The aim of the study was to identify predictors of early-onset neonatal infections.

Material and methods. We conducted a prospective study at the Neonatology Clinic within the County Clinical Hospital in Tg. Mures, Romania, between January 2017 and March 2019.

Results. The study included 120 newborns, of which 15 (12.5\%) had an APGAR score of 10 per 1 minute. At 5 minutes, $57(47.5 \%)$ had an APGAR score of 10 . Type of birth: 59 (49.2\%) of the patients were born vaginally and $61(50.8 \%)$ by cesarean section. The average of the gestational age was 39.04 weeks. The average weight at birth was 3,197.33 g, and at discharge was $3,126.12 \mathrm{~g}$. The average length of the new-borns was $51.62 \mathrm{~cm}$, and the average of the cranial perimeter was $33.48 \mathrm{~cm}$. Most frequently, patients presented infectious pathologies, early neonatal $(n=40)$, frequently associated with the damage of the lung system. The average length of time spent in hospital was of 4 days, with an average of 6.15 days. The APGAR score at 1 minute and 5 minutes was statistically significantly correlated with the number of days of hospitalization and parity of the mother.

Conclusions. The APGAR score at 1 minute and 5 minutes was the most accurate indicator for the risk of neonatal infection in our study. Its values correlated with the number of days of hospitalization. The number of hospitalization days was also correlated with parity.
\end{abstract}

Keywords: early neonatal infections, APGAR score, C-reactive protein

\section{INTRODUCTION}

Infections in newborns are a widely studied topic worldwide. The increased vulnerability of newborns to infections compared to other ages has been demonstrated at both the cellular and molecular levels $(1,2)$. Immaturity and inexperience of the innate and adap- tive immune system make newborns, especially those born prematurely, much more susceptible to infections, both bacterial and viral (3).

The pathophysiological mechanisms involved in the development of neonatal infections are not fully understood. It appears that immune immaturity and virulence of microbial agents, partly explain the dif- 
ferences between infected infants with the same pathogens, frequently acquired in the neonatal period. The discovery of the role of maternal antibodies (Abs) such as streptococcal group B anti-capsular polysaccharides (GBS) - in protecting newborns against neonatal sepsis was a milestone in discovering the molecular basis of the susceptibility to neonatal infection (1).

Neonatal infections are divided into early-onset, acquired infections with microorganisms from the female genital tract, most often caused by ascending amniotic infection or peripartum, and those with lateonset, which occur as a result of invasive procedures due nosocomial germs or peri or postpartum invasion. Susceptibility to infection is aggravated as a result to the relatively immaturity immunity, and the incidence of infection is inversely proportional to gestational age and birth weight (BW) $(4,5)$.

Globally, the World Health Organization (WHO) estimates that neonatal deaths represent $44 \%$ of global deaths, of which $26 \%$ are caused by infections $(6,7)$.

Acquisition of the infection can take place at any time: ante, per or postnatal. Postnatal infections can be transmitted through direct contact with hospital staff, mothers, family members, breastfeeding or various contaminated surfaces (8).

Another very important aspect in the evolution of infections in newborns is sepsis. Neonatal sepsis is a major cause of morbidity and mortality worldwide. In 2015 , infections were responsible for $15 \%$ of the 2.7 million neonatal deaths globally. It is estimated that 150,000 babies die from sepsis on the first day after birth and 300,000 by the end of the first week (9). Globally, of the three million annual cases of neonatal sepsis $(2,202 / 1,000,000$ live births), India has the highest incidence of clinical sepsis $(17,000 / 1,000,000$ live births) (10).

In the early 1950s, in America, Virginia Apgar developed the APGAR score, currently used, to assess the health of the newborn. The 5 parameters on the basis of which the APGAR index is assessed are: respiration, heart rate, reactivity to stimuli, muscle tone, skin appearance - parameters that will be marked each with points from 0 to 2 , so that the score can be between 0 and 10 (11).

\section{OBJECTIVE}

The aim of the study was to identify predictive factors for early-onset neonatal infections.

\section{MATERIAL AND METHODS}

We conducted a prospective study at the Neonatology Clinic within the County Clinical Hospital in Târgu Mureș, Romania, between January 2017 and March 2019, which included 120 newborns. Informed consent from parents or legal guardians was obtained for each patient.

The variables studied were the APGAR score at 1 minute and 5 minutes, type of birth, weight at childbirth and at discharge, hospitalization diagnoses, complete blood count, antibiotic therapy, number of days of hospitalization and patient evolution. Data were extracted from patient records.

Analytical statistics were performed using SPSS v17.0 software, and GraphPad Prism 8 and XLSTATLite 2015 software for Windows 10 Pro were used for descriptive statistics. The Grubbs test was used to eliminate aberrant values. The Kolmogorov-Smirnov test was used to identify the Gaussian distribution of data; for the parametric data the Pearson test was applied, respectively the Spearman test for the non-parametric data. The results were considered statistically significant for $\mathrm{p}$ values less than 0.05 .

The study was conducted according to the principles of the Helsinki Declaration and was approved by the Ethics Commission of the "George Emil Palade” University of Medicine, Pharmacy, Science and Technology, Tg. Mures no. 71/27.4.2018.

\section{RESULTS}

The study included 120 newborns, 52 (43.3\%) females and 68 (56.7\%) males; of which 8 were included in the category of social cases. $15(12.5 \%)$ of the 120 patients included in the study had a score of 10 on the APGAR score at 1 minute. At 5 minutes, 57 patients $(47.5 \%)$ had a score of 10 . Regarding the type of birth and gestational age, 59 (49.2\%) patients were born vaginally and $61(50.8 \%)$ by cesarean section; the average gestational age being 39.04 weeks. The average weight at birth was $3,197.33 \mathrm{~g}$ and at discharge was $3,126.12 \mathrm{~g}$. The average length of the fetus was $51.62 \mathrm{~cm}$, and the average of the cranial perimeter was $33.48 \mathrm{~cm}$.

Most frequently the patients presented infectious pathologies $(n=40)$, frequently associated with the damage of the lung system. The incidence of diseases depending on the organ systems at birth is represented in Figure 1. 


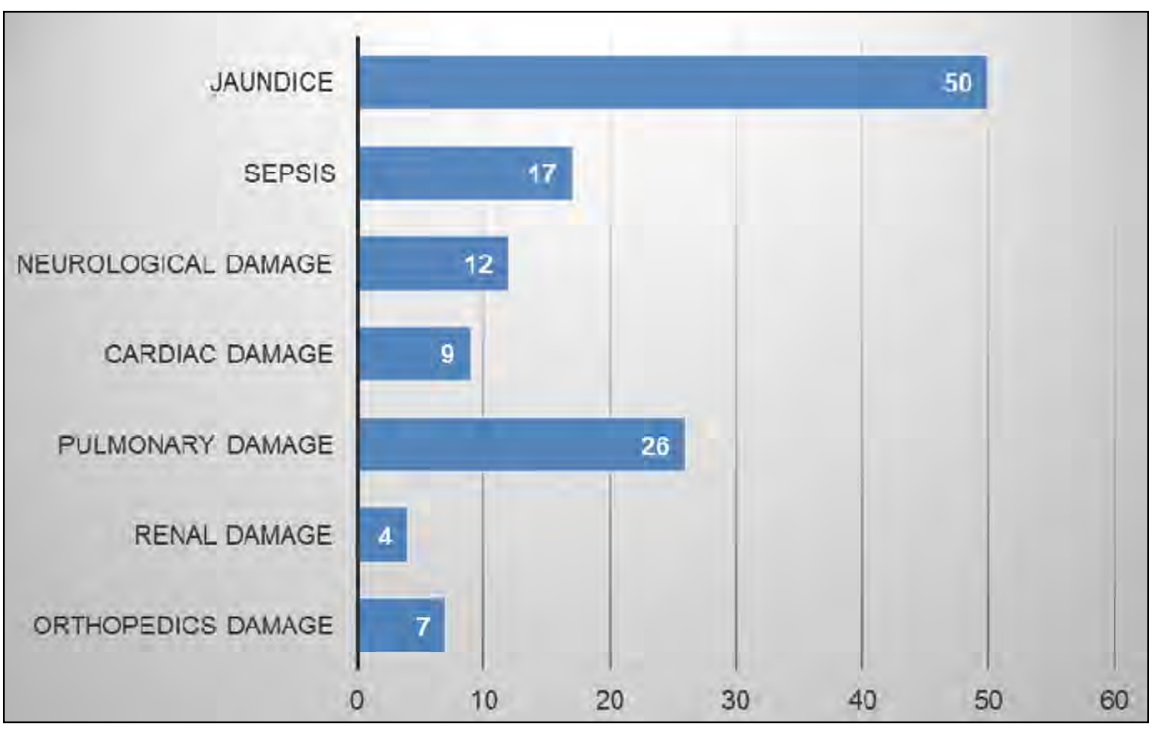

FIGURE 1.The incidence of the diseases based on organ systems

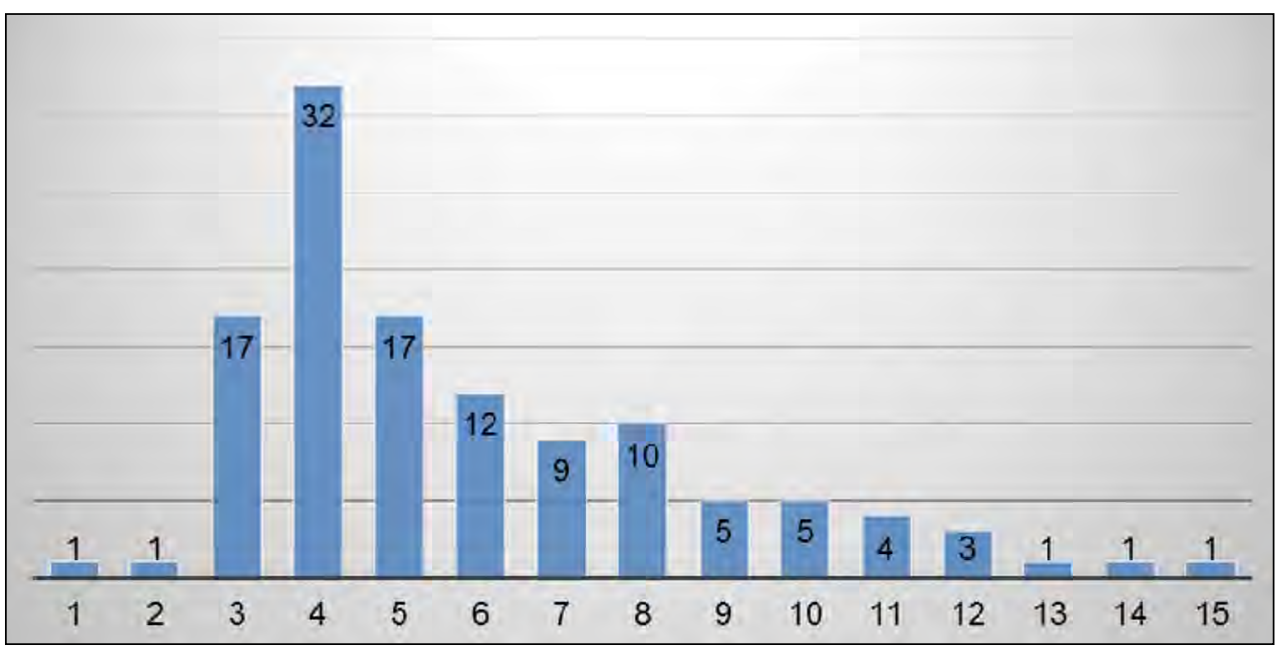

FIGURE 2. Distribution of cases by number of days of hospitalization

Early neonatal infection was present in the 40 newborns with infectious pathology. Most of them were hospitalized for 4 days, with an average of 6.15 days. The distribution of patients by hospitalization days was performed in Figure 2.

In the study, the correlations between the values of the parameters obtained from the complete blood count (hemoglobin, hematocrit, leukocytes, lymphocytes, neutrophils, monocytes and platelets) and the number of hospitalization days, the APGAR score at one minute and at five minutes and the values of $\mathrm{C}$ reactive protein were analyzed.

In our study, we obtained an average of C-reactive protein values ollected in the first 24 hours of hospitalization, among newborns with infections of 8.87 $\mathrm{mg} / \mathrm{dl}$. We considered this value a predictive factor in terms of the infectious status of newborns.
Among the analyzed parameters, we obtained a negative statistically significant correlation between the APGAR score level at one minute $(\mathrm{p}=0.0054, \mathrm{r}=$ $-0.2558)$ and at five minutes $(p=0.0003, r=-0.3276)$, in relation with the days of hospitalization. Also, for parity of the mother, in relation with the days of hospitalization, we obtained a negative correlation statistically significant $(\mathrm{p}=0.0426, \mathrm{r}=-0.1894)$.

Following the implementation of the Chi ${ }^{2}$ test, a statistically significant dependency relationship was identified $(\mathrm{p}=0.0264)$ between the APGAR score at 1 minute and the infectious status of the patients.

Of the 120 patients included in the study, 50 (41.66\%) received antibiotic treatment, of which 10 patients $(12 \%)$ received prophylactic treatment. 33 (27.5\%) of the patients received Ampiplus together with Amikacin or Gentamicin; 15 (12.5\%) received 
TABLE 1. Statistical correlations for APGAR score (1, and 5')

\begin{tabular}{|c|c|c|c|c|c|c|c|c|}
\hline \multirow{2}{*}{ Analyzed variables } & \multicolumn{2}{|c|}{ APGAR $1^{\circ}$} & \multicolumn{2}{|c|}{ APGAR $5^{\prime}$} & \multicolumn{2}{|c|}{ Gravidity } & \multicolumn{2}{|c|}{ Parity } \\
\hline & $n$ & $p$ & $n$ & $p$ & $n$ & $p$ & $n$ & $p$ \\
\hline \multirow{2}{*}{ Hospitaliza } & \multirow{2}{*}{119} & 0.0054 & \multirow{2}{*}{119} & 0.0003 & \multirow{2}{*}{118} & \multirow{2}{*}{0.3652} & \multirow{2}{*}{119} & 0.0426 \\
\hline & & $r=-0.2558$ & & $r=-0.3276$ & & & & $r=-0.1894$ \\
\hline Birth weight & 120 & 0.4382 & 120 & 0.2246 & 118 & 0.5012 & 116 & 0.79603 \\
\hline
\end{tabular}

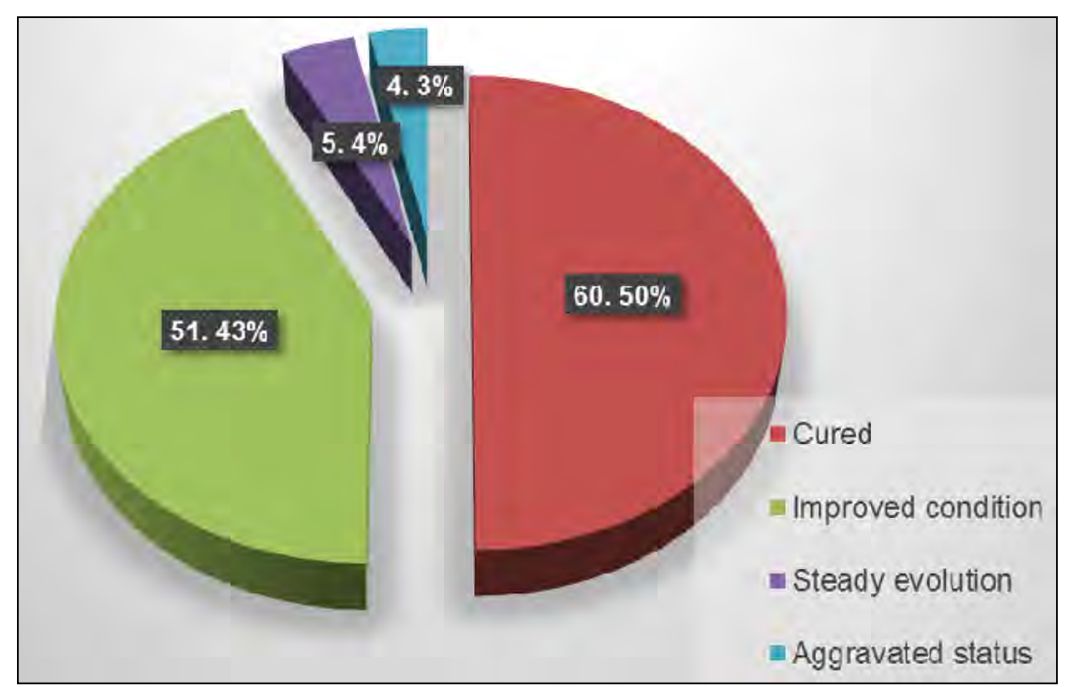

FIGURE 3. Patients' discharge status

Ampicilin together with Gentamicin. Of these, 19 patients (38\%) recovered and $25(50 \%)$ had an improved condition.

At discharge, $60(50 \%)$ patients were cured, 51 (42.5\%) had an improved condition, 5 (4.16\%) had a steady evolution, and $4(3.33 \%)$ had a aggravated status (Figure 3).

\section{DISCUSSIONS}

Neonatal infection is one of the most important causes influencing the rate of mortality and morbidity in newborns. Low birth weight, anemia and young age of the mother are considered to be important risk factors in terms of the infectious status of the newborn. The severity of the infection in newborns is given by the fact that it can easily progress to sepsis, which represents $10 \%$ of the causes of mortality in newborns, currently being one of the biggest challenges in the field of neonatology $(12,13)$.

In the literature, neonatal infection is subclassified into early-onset infection, occurring at less than 72 hours after birth, and late-onset infection at more than 72 hours after birth (14). Although late-onset neonatal infection is much more common than early-onset infection, the latter one has a more aggressive course, with a more reserved prognosis. The worldwide inci- dence of late-onset neonatal infection is of 61 per 1,000 newborns, respectively of early-onset neonatal infection is of 9 per 1,000 newborns. Most cases of early-onset infections (80-90\%) occur in the first 48 hours of life (15). In our study, we followed the evolution of 40 newborns included in the study group $(\mathrm{n}=$ 120), who developed infections in the first 72 hours after birth. Another difference in terms of pathogens identified in each type of neonatal infection is that in late-onset neonatal infection are found Streptococcus type B (in 54\% of cases), Enterobacteriaceae (21\%) and Staphylococcus aureus (18\%), and in the case of early-onset infections, Streptococus agalactiaei belonging to group B, representing 58\%, and Escherichia coli with $18 \%$ of infections are found. Agents that cause congenital or perinatal infections can lead to permanent hearing loss, vision loss, cognitive impairment, cerebral palsy, developmental delay, behavioral disorders, or epilepsy. Carrying out blood culture and identifying the pathogen are essential in establishing the appropriate and effective treatment.

Knowledge of maternal symptoms and history, as well as screening results for maternal infections in the last trimester of pregnancy should also be considered. Usually, Ampicillin in combination with an aminoglycoside are administered in the treatment of these infections, they are being considered broad-spectrum 
antibiotics, covering infections with Streptococcus, E. coli or other common pathogens (16-18). This correlates with the data from our study, where in most cases Ampiplus was administered with Amikacin or Gentamicin, the last two belonging to the class of aminoglycosides.

International recommendations indicate 7-10 days of treatment in the presence of microbiologically confirmed bacterial infections or in the case of unconfirmed ones, but with a strong clinical presentation. In the presence of a negative blood culture test after 3648 hours and in the absence of a strong clinical suspicion of neonatal infection, interruption of antibacterial therapy should be considered. The scientific literature does not recommend any other lenght of treatment and, therefore, a lenght of antibiotic therapy of 5 or 6 days raises doubts about the effectiveness of antibiotic therapy in such circumstances. The average number of days of hospitalization in our study at patients with infection is 8.4 days, this coincides in most cases with the number of days of antibiotic administration. Medical treament compliance is also observed in the fact that only 4 of the 50 newborns who underwent antibiotic therapy had an unfavorable evolution $(19,20)$. Respiratory distress is the accumulation of fluid in the lungs that causes difficulty in breathing. It is common in the first stage in newborns, especially those born after cesarean section and is often remedied in just a few hours. Newborns considered stable and with no perinatal risk factors for developing sepsis may be monitored for up to 6 hours to determine if respiratory function syndrome resolves before continuing sepsis investigations and initiating antibiotic therapy (21). In our study, it was present in 10 newborns, and 6 of them had a serious evolution towards aspiration pneumonia, respectively sepsis. All underwent antibiotic treatment.

In some publications, the association between normal C-reactive protein (CRP) levels in serological tests with a significantly lower probability of infection has been reported, with probability ratios ranging from 0.03 to 0.16 . Maximum PCR levels greater than $3 \mathrm{mg} / \mathrm{dl}$ have positive predictive values greater than $20 \%$ for early and late onset infections, but only those with values above $6 \mathrm{mg} / \mathrm{dl}$ have a very high positive predictive value for neonatal infections, respectively sepsis with early onset (6). Blood levels of C-reactive protein are useful in the diagnostic evaluation of the newborns who are suspected of having an infectious syndrome. Previous studies have shown that two lev- els of C-reactive protein less than $1 \mathrm{mg} / \mathrm{dl}$ obtained 24 hours apart, between 8 to 48 hours after presentation, indicate that bacterial infection is unlikely. The sensitivity of a normal C-reactive protein value at the initial assessment is not sufficient to justify the lack of antibiotic therapy $(22,23)$.

In our study we obtained a statistically significant relationship between the APGAR score (1 minute and 5 minutes) and the infectious status of newborns. The relationship between APGAR score less than 7 at birth and infant mortality is mainly attributed to anoxia or infections, but a relatively normal APGAR score between 7 and 9 may be followed by the presence of a neonatal infection and may also be associated with high level risks of neonatal mortality and morbidity. The lower the APGAR score is at 5 minutes, the greater is the risk. The strong relationships between APGAR scores of 7, 8, and 9 and neonatal morbidity observed in our study provide insight into the increased risk of infections at these values compared to a score of 10 at 1 minute and 5 minutes.

Therefore, more and more studies are focusing on the prophylaxis required for an APGAR score of 10 (24). Similar to the data in the literature, we obtained in our study the fact that of the 15 (18\%) newborns included in the study who obtained the APGAR score 10 at 1 minute, none suffered from infectious pathologies and required the fewest days of hospitalization. In 10 patients $(21.2 \%)$ of the 47 newborns who were initially evaluated with an APGAR score of less than 10 , between 7 and 9 at one minute and subsequently at 5 minutes, obtained an APGAR score of 10 , suffered from infectious pathologies.

The most important limitation of the study was the relatively small number of patients $(n=120)$. Other limitations: we analyzed data from a single neonatology clinic and we did not include in the study premature newborns, who were treated in another center.

\section{CONCLUSIONS}

The most common pathology encountered in newborns was infectious. The APGAR score at one minute and at five minutes was the most accurate indicator for the risk of neonatal infection in our study. Its values were also correlated with the number of days of hospitalization. Also, the number of days of hospitalization was correlated with parity. The treatment followed by most patients was the combination of a beta-lactam and an antibiotic from the class of aminoglycosides. 


\section{REFERENCES}

1. Borghesi A, Stronati M, Castagnoli R et al. Novel Approaches to the Study of Neonatal Infections. Am J Perinatol. 2018;35(6):570-574.

2. Levy O. Innate immunity of the newborn: Basic mechanisms and clinical correlates. Nat Rev Immunol. 2007;7(05):379-390.

3. Perez GF, Pancham K, Huseni S, Jain A, Rodriguez-Martinez CE, Preciado $D$ et al. Rhinovirus-induced airway cytokines and respiratory morbidity in severely premature children. Pediatr Allergy Immunol. 2015;26:145-52.

4. Darlow BA, Voss L, Lennon DR et al. Early-onset neonatal group B streptococcus sepsis following national risk-based prevention guidelines. Aust N Z J Obstet Gynaecol. 2016;56:69-74.

5. Singh T, Barnes EH, Isaacs D; Australian Study Group for Neonatal Infections. Early-onset neonatal infections in Australia and New Zealand, 2002-2012. Arch Dis Child Fetal Neonatal Ed. 2019; 104(3):F248-F252.

6. Darmstadt GL, Marchant $\mathrm{T}$, Claeson $\mathrm{M}$ et al. A strategy for reducing maternal and newborn deaths by 2015 and beyond. BMC Pregnancy Childbirth. 2013;13:216.

7. World Health Organization. World Health Report 2005: Make Every Mother and Child Count. Geneva: World Health Organization; 2005.

8. Yoon HS, Shin YJ, Ki M. Risk factors for neonatal infections in full-term babies in South Korea. Yonsei Med J. 2008;49(4):530-536.

9. UNICEF. The neonatal period is the most vulnerable time for a child. UNICEF, Neonatal Mortality, 2016. https:// data. unicef. org/ topic/ child- survival/ neonatal- mortality/ (accessed 21 Aug 2017).

10. Fleischmann-Struzek C, Goldfarb DM, Schlattmann P, Schlapbach LJ, Reinhart K, Kissoon N. The global burden of paediatric and neonatal sepsis: A systematic review. Lancet Respir Med. 2018; 6(3):223-230.

11. Cnattingius S, Norman M, Granath F, Petersson G, Stephansson O, Frisell T. Apgar Score Components at 5 Minutes: Risks and Prediction of Neonatal Mortality. Paediatr Perinat Epidemiol. 2017; 31(4):328-337.

12. Simonsen KA, Anderson-Berry AL, Delair SF, Davies HD. Early-onset neonatal sepsis. Clin Microbiol Rev. 2014;27(1):21-47.

13. Lim JW, Chung SH, Kang DR, Kim CR. Risk Factors for Cause-specific Mortality of Very-Low-Birth-Weight Infants in the Korean Neonatal Network. J Korean Med Sci. 2015.
14. Haque K. Definitions of bloodstream infection in the newborn. Pediatr Crit Care Med. 2005;6(3 Suppl):S45-9.

15. National Institute for Health and Care Excellence (NICE). Antibiotics for neonatal infection. NICE quality standard 75. 2014; guidance. nice.org.uk/qs75, p.5.

16. National Collaborating Centre for Women's and Children's Health. Antibiotics for early-onset neonatal infection: antibiotics for the prevention and treatment of early-onset neonatal infection. Royal College of Obstetricians and Gynaecologists. 2012.

17. Maródi L. Neonatal innate immunity to infectious agents. Infect Immun. 2006 Apr;74(4):1999-2006.

18. Velaphi S, Siegel JD, Wendel GD Jr, Cushion N, Eid WM, Sanchez PJ. Early-onset group B streptococcal infection after a combined maternal and neonatal group B streptococcal chemoprophylaxis strategy. Pediatrics. 2003;111(3):541-7.

19. NICE clinical guideline. Antibiotics for early-onset neonatal infection Antibiotics for the prevention and treatment of earlyonset neonatal infection. Available from: http://www.nice.org.uk/ guidance/CG149, date of publication: August 2012.

20. Escobar GJ, Puopolo KM, Wi S et al. Stratification of risk of early-onset sepsis in newborns $\geq 34$ weeks' gestation. Pediatrics. 2014;133(1):30-36.

21. Brown JVE, Meader N, Wright K, Cleminson J, McGuire W. Assessment of C-Reactive Protein Diagnostic Test Accuracy for Late-Onset Infection in Newborn Infants: A Systematic Review and Meta-analysis [published online ahead of print, 2020 Feb 3]. JAMA Pediatr. 2020;174(3):260-268.

22. Ahmed E, Rehman A, Ali MA. Validation of serum C-reactive protein for the diagnosis and monitoring of antibiotic therapy in neonatal sepsis. Pak J Med Sci. 2017;33(6):1434-1437.

23. Gutbir $Y$, Wainstock $T$, Sheiner $E$ et al. Low Apgar score in term newborns and long-term infectious morbidity: A population-based cohort study with up to 18 years of follow-up. Eur $J$ Pediatr. 2020; 179(6):959-971.

24. Razaz N, Cnattingius S, Joseph KS. Association between Apgar scores of 7 to 9 and neonatal mortality and morbidity: Population based cohort study of term infants in Sweden. BMJ 2019;365:I1656.

Conflict of interest: none declared Financial support: none declared 